\title{
Contributions to the knowledge of amphibians and reptiles from Volta Grande do Xingu, northern Brazil
}

\author{
Vaz-Silva, W. ${ }^{*}$, Oliveira, RM. ${ }^{b}$, Gonzaga, AFN. ${ }^{b}$, Pinto, KC. ${ }^{b}$, Poli, FC. ${ }^{b}$, Bilce, TM. ${ }^{b}$, \\ Penhacek, M. ${ }^{b}$, Wronski, L. ${ }^{b}$, Martins, JX. ${ }^{b}$, Junqueira, TG. ${ }^{b}$, Cesca, LCC. ${ }^{b}$, Guimarães, VY. ${ }^{b}$ \\ and Pinheiro, RD. ${ }^{b}$
}

aPrograma de Pós-Graduação em Ciências Ambientais e Saúde, Departamento de Biologia, Centro de Estudos e Pesquisas Biológicas, Pontifícia Universidade Católica de Goiás - PUC Goiás, Rua 235, 40, Bloco L, Setor Universitário, CEP 74605-010, Goiânia, GO, Brazil

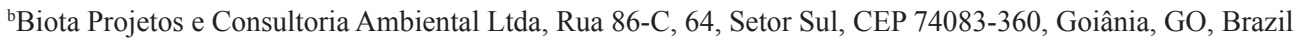
*e-mail: herpetovaz@gmail.com

Received: June 2, 2014 - Accepted: October 8, 2014 - Distributed: August 31, 2015

(With 5 figures)

\begin{abstract}
The region of Volta Grande do Xingu River, in the state of Pará, presents several kinds of land use ranging from extensive cattle farming to agroforestry, and deforestation. Currently, the Belo Monte Hydroelectric Power Plant affects the region. We present a checklist of amphibians and reptiles of the region and discuss information regarding the spatial distribution of the assemblies based on results of Environmental Programmes conducted in the area. We listed 109 amphibian (Anura, Caudata, and Gymnophiona) and 150 reptile (Squamata, Testudines, and Crocodylia) species. The regional species richness is still considered underestimated, considering the taxonomic uncertainty, complexity and cryptic diversity of various species, as observed in other regions of the Amazon biome. Efforts for scientific collection and studies related to integrative taxonomy are needed to elucidate uncertainties and increase levels of knowledge of the local diversity.
\end{abstract}

Keywords: herpetofauna, monitoring, Amazonia, Belo Monte.

\section{Contribuições para o conhecimento de anfíbios e répteis da Volta Grande do Xingu, norte do Brasil}

\begin{abstract}
Resumo
A região da Volta Grande no Rio Xingu, localizada no estado do Pará, apresenta várias frentes de exploração que vão desde a criação animal até área de cultivo, resultando no desmatamento de fragmentos florestais. Atualmente a região está sendo impactada pela implantação da Usina Hidrelétrica de Belo Monte. A partir de dados obtidos através dos Programas de Monitoramento Ambiental realizados na área de influência da UHE Belo Monte, apresentamos uma listagem das espécies de anfíbios e répteis, com comentários sobre a distribuição espacial das assembleias. Foram catalogados 109 espécies de anfíbios (Anura, Caudata e Gymnophiona) e 150 de répteis (Squamata, Testudines e Crocodylia). Considerando as incertezas taxonômicas, a complexidade e a diversidade críptica de várias espécies, a riqueza regional ainda continua subestimada, assim como observado em outras regiões da Amazônia. Esforços direcionados ao aproveitamento científico e estudos que contemplem a taxonomia integrativa são necessários para elucidar as incertezas e aumentar o nível de conhecimento sobre a diversidade da região.
\end{abstract}

Palavras-chave: herpetofauna, monitoramento, Amazônia, Belo Monte.

\section{Introduction}

Amazonia is the largest tropical rainforest in the world and provides important ecosystem services as well high biodiversity and climate regulation (Coe et al., 2013). Azevedo-Ramos and Galatti (2002) presented a survey of information on amphibian richness in the Brazilian Amazon, estimating a minimum of 163 species. Ávila-Pires et al. (2007) report the occurrence of 232 species of amphibians and 273 species of reptiles. This richness is underestimated considering the taxonomic problems (Funk et al., 2011), the recent descriptions of species (e.g. Sturaro and Peloso, 2014) and taxonomic revisions (e.g. Maciel and Hoogmoed, 2011; Brcko et al., 2013).

There is a lack of information of the current status of the Brazilian Amazonian Herpetofauna due to dispersed 
and unpublished data (Azevedo-Ramos and Galatti, 2002; Ávila-Pires et al., 2007). Knowledge has increased in the last 10 years based on studies conducted in the Manaus region (e.g. Lima et al., 2006, Menin et al., 2007, Vitt et al., 2008), State of Pará (e.g. Frota, 2004; Caldwell and Araújo, 2005; Frota et al., 2005; Prudente and Santos-Costa, 2005; Ávila-Pires et al., 2009; Maschio et al., 2009; Mendes-Pinto and Tello, 2010; Bitar et al., 2011; Frota et al., 2011; Mendes-Pinto and Souza, 2011), State of Rondônia (e.g. Bernarde and Abe, 2006; Bernarde, 2007; Bernarde and Macedo, 2008; Macedo et al., 2008; Avila-Pires et al., 2009) and in the State of Acre (e.g. Souza et al., 2003; Souza et al., 2008; Bernarde et al., 2011).

Faunal inventories are important tools for Amazonian conservation, since biological surveys stimulate the discovery of new species, revealing endemic areas and the current levels of biodiversity (França and Venâncio, 2010). The production of scientific knowledge resulting from activities related to environmental projects when carried out by specialist teams is one of the most important ways to mitigate the impacts generated by impactful project implementation (Vaz-Silva, 2009). The knowledge generated is essential for making decisions and the establishment of conservation policies.

The aim of this paper is to present the current status of knowledge of the amphibians and reptiles from Volta Grande do Xingu, in the state of Pará, based on information obtained after three years of the Faunal Rescue Programme and two years of the Environmental Monitoring Programme in the region of influence of the Belo Monte Hydroelectric Power Plant (UHE Belo Monte). Also discussed will be information concerning the spatial distribution of the assemblies in the monitored sites.

\section{Material and Methods}

The UHE Belo Monte is located in Volta Grande do $\mathrm{Xingu}$, on the right bank tributary of the Amazon River, state of Pará, northern Brazil (Figure 1). The Hydroelectric project consists of the dam, reservoir, water intake and power house, occupying part of Altamira, Vitória do Xingu, Anapu, and Brasil Novo municipalities. The basin of Rio Xingu, with a total area of $509,000 \mathrm{~km}^{2}$, has not suffered greatly from deforestation. The area of the UHE Belo Monte, due to its proximity to the regional centre of Altamira city and

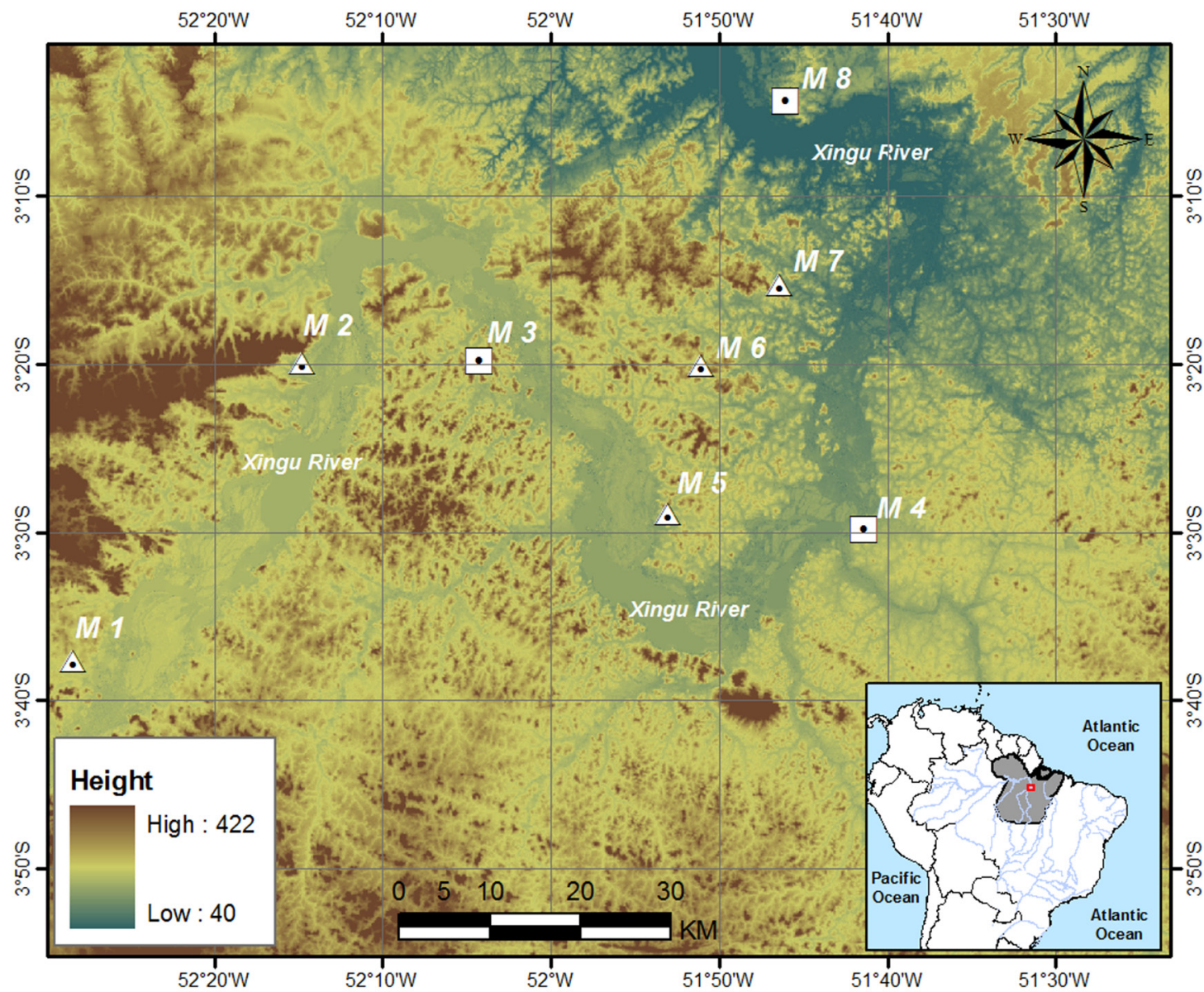

Figure 1. Hypsometric map showing the location of the study site and the altitude variation (height), Volta Grande do Xingu River. M1 - M8 = modules RAPELD 1 to 8. 
highway BR-230 (Transamazônica), has many uses ranging from extensive cattle farming to agroforestry, and natural forest remains (Souza Junior et al., 2006). The vegetation types in the region are the Floresta Ombrófila Densa (Dense Rain Forest), Floresta Ombrófila Densa Aluvial (Alluvial Rain Forest), Open Rain Forest with Palm Trees and Vines (Floresta Ombrófila Aberta com Cipós e Palmeiras) (Salomão et al., 2007).

We present the results of the Environmental Impact Study (EIA-RIMA) (Leme Engenharia, 2009) and preliminary data of the Environmental Monitoring Programmes conducted during the installation of the Belo Monte Hydroelectric Power Plant (Faunal Rescue Programme and Environmental Monitoring Programme of Herpetofauna). We compare the presented results with the regional knowledge based on published papers (Caldwell and Araújo, 2005; Oliveira et al., 2013) to produce the checklist of the species.

The EIA-RIMA considered three field expeditions between 2000-2001 and 2007-2008 (Leme Engenharia, 2009). The Faunal Rescue Programme contemplated management actions implemented during the suppression of vegetation in the area of direct influence of the UHE Belo Monte from June 2011 to April 2014. We considered only standardised data from the Environmental Monitoring Programme of Herpetofauna for analyses. The analyses grouped results from five expeditions (2011 to 2014), with samples obtained from eight sampling sites (modules M1 to M8), considered as the RAPELD methodology (Magnusson et al., 2005, 2013). Voucher specimens are housed at the Museu Paraense Emílio Goeldi (Belém), Museu Nacional (Rio de Janeiro), Museu de Zoologia da Universidade de São Paulo (São Paulo), and at the Coleção Herpetológica da Universidade Federal de Goiás (Goiânia).

We calculated the dissimilarity matrix for the modules (M1 to M8) considering the abundance of species, using the coefficient Bray Curtis. Cluster analysis was performed using the method of unweighted average linkage (UPGMA) from the Bray-Curtis dissimilarity matrix using Past software (Hammer et al., 2001). Diversity between sampling sites was compared by Hill's series (Hill, 1973): $\mathrm{N} \_\mathrm{a}=\left(\mathrm{p} \_1^{\wedge} \mathrm{a}+\mathrm{p} \_2^{\wedge} \mathrm{a}+\ldots+\mathrm{p} \_\mathrm{n}^{\wedge} \mathrm{a}\right)^{\wedge}(1 /(1-\mathrm{a}))$. Where: $N_{a}$ is the diversity index value by parameter $a$; $n$ is the number of species; and, $p_{i}$ proportional abundance of the species $i$. Hill's series was calculated by R software 3.0.1 (R Development Core Team, 2013), vegan package (Oksanen et al., 2013). The taxonomic arrangement used in the checklist followed Pyron et al. (2013) and Frost (2014).

\section{Results and Discussion}

The cumulative data of the Environmental Programmes from earlier times before the operation of the UHE Belo Monte resulted in a checklist of 109 amphibian and 150 reptile species (Table 1 ). Volta Grande do Xingu corresponds to a region with few Herpetofaunal inventories. Some ecological, population and natural history studies of the chelonians, amphibians and lizards have been conducted in the region (Caldwell and Lopez, 1989; Howland et al., 1990; Vitt and Blackburn, 1991; Vitt and Breitenbach, 1993; Vitt et al., 1997; Vitt and Zani 1998; Pearse et al., 2006). Caldwell and Araújo (2005) reported the occurrence of 40 species of amphibians from Cachoeira Juruá. Oliveira et al. (2013) reported the occurrence of 30 species of amphibians, 13 snakes and 7 lizard species from the municipality of Brasil Novo municipality. Knispel and Barros (2009) reported the occurrence of 15 species of amphibians for the urban area of Altamira. Maciel et al. (2013) reported the occurrence of Siphonops anullatus in the municipality of Senador Jose Porfírio, showing the incipient knowledge of the local herpetofauna.

According to the EIA-RIMA, 60 amphibian and 87 reptile species are found in the region of the UHE Belo Monte, and this richness can reach 68 amphibians and 118 reptiles considering the regional knowledge. The increased sampling effort provided by Environmental Monitoring Programmes from the UHE Belo Monte resulted in the increase of 111 species (48 amphibians and 63 reptiles, including those with open nomenclature) to the Herpetofauna checklist (Table 1). The cumulative data show a high diversity compared to other sites of the Amazonian biome (17 amphibian and 40 reptilian at Cacoal - Turci and Bernarde, 2008; 56 amphibian and 53 reptilian species at Boca do Acre, state of Amazonas - França and Venâncio, 2010; 83 amphibian and 79 reptilian at Reserva Extrativista Riozinho da Liberdade - Bernarde et al., 2011; 35 amphibian and 59 reptilian species at Floresta Nacional do Trairão - Mendes-Pinto and Souza, 2011; 59 reptilian species at Barcarena - Silva et al., 2011; 38 reptilian species at Alto Alegre dos Parecis - Ferrão et al., 2012; 71 amphibian species at Carajás region - Pinheiro et al., 2012; and 46 amphibian and 38 reptilian species at Reserva Extrativista do rio Gregório - Pantoja and Fraga, 2012).

New records in the state (e.g. Frota and Vaz-Silva, 2013; Camera and Krinski, 2014) evidence the incipience of knowledge in the regional context due to the lack of sampling. The checklist presented represents an increase in regional knowledge. The records of sympatric Centrolenidae species (this study) are important considering the ecological aspects of the species. Centrolenids are considered specialists in the use of the environment, occurring on the banks of streams and waterfalls in woody vegetation where they lay their egg masses (Cisneros-Heredia and Mcdiarmid, 2007). Considering the impact resulting from the implementation of the UHE Belo Monte, which will result in the suppression of habitats associated with the occupation of Centrolenids, monitoring of these populations is an important factor in the continuity of the Monitoring Programme.

Recent studies evidence that species of this family are distributed throughout the Amazon basin, as reported for Cochranella adenocheira by Toledo et al. (2009) and Oliveira et al. (2012), Hyalinobatrachium iaspidiensis by Yanez-Muñoz et al. (2009), Hyalinobatrachium cappellei by Simões et al. (2012), Hyalinobatrachium caslesvilai by Cisneros-Heredia et al. (2010). Endemics restricted in the biome can be attributed to the lack of sampling. Other 
Table 1. Checklist of amphibians and reptiles from Volta Grande do Xingu, state of Pará, Brazil. EIA-RIMA= Environmental Impact Study; FRF = Faunal Rescue Programme; MEP = Monitoring Environmental Programme.

\begin{tabular}{|c|c|c|c|}
\hline Taxon & EIA-RIMA & FRP & MEP \\
\hline \multicolumn{4}{|l|}{ Class Amphibia } \\
\hline \multicolumn{4}{|l|}{ Family Bufonidae } \\
\hline Amazophrynella cf. bokermanni (Izecksohn, 1994 “1993”) & $\mathrm{X}$ & & $\mathrm{X}$ \\
\hline Rhaebo guttatus (Schneider, 1799) & $\mathrm{X}$ & $\mathrm{X}$ & $\mathrm{X}$ \\
\hline Rhinella castaneotica (Caldwell, 1991) & & $\mathrm{X}$ & $\mathrm{X}$ \\
\hline Rhinella major (Muller and Helmich, 1936) & $\mathrm{X}$ & $\mathrm{X}$ & $\mathrm{X}$ \\
\hline Rhinella margaritifera (Laurenti, 1768) & $\mathrm{X}$ & & $\mathrm{X}$ \\
\hline Rhinella marina (Linnaeus, 1758) & $\mathrm{X}$ & $\mathrm{X}$ & \\
\hline \multicolumn{4}{|l|}{ Family Ceratophryidae } \\
\hline Ceratophrys cornuta (Linnaeus, 1758) & $\mathrm{X}$ & $\mathrm{X}$ & $\mathrm{X}$ \\
\hline \multicolumn{4}{|l|}{ Family Odontophrynidae } \\
\hline Proceratophrys sp. & $\mathrm{X}$ & $\mathrm{X}$ & $\mathrm{X}$ \\
\hline \multicolumn{4}{|l|}{ Famíly Leptodactylidae } \\
\hline Adenomera cf. andreae (Müller, 1923) & & $\mathrm{X}$ & $\mathrm{X}$ \\
\hline Adenomera hylaedactyla (Cope, 1868) & & $\mathrm{X}$ & $\mathrm{X}$ \\
\hline Adenomera sp. & $\mathrm{X}$ & & $\mathrm{X}$ \\
\hline Leptodactylus knudseni Heyer, 1972 & & $\mathrm{X}$ & $\mathrm{X}$ \\
\hline Leptodactylus leptodactyloides (Andersson, 1945) & $\mathrm{X}$ & & $\mathrm{X}$ \\
\hline Leptodactylus longirostris Boulenger, 1882 & & $\mathrm{X}$ & $\mathrm{X}$ \\
\hline Leptodactylus macrosternum Miranda-Ribeiro, 1926 & $\mathrm{X}$ & & $\mathrm{X}$ \\
\hline Leptodactylus mystaceus (Spix, 1824) & $\mathrm{X}$ & $\mathrm{X}$ & $\mathrm{X}$ \\
\hline Leptodactylus paraensis Heyer, 2005 & $\mathrm{X}$ & $\mathrm{X}$ & $\mathrm{X}$ \\
\hline Leptodactylus pentadactylus (Laurenti, 1768) & $\mathrm{X}$ & $\mathrm{X}$ & $\mathrm{X}$ \\
\hline Leptodactylus petersii (Steindachner, 1864) & $\mathrm{X}$ & $\mathrm{X}$ & $\mathrm{X}$ \\
\hline Leptodactylus podicipinus (Cope, 1862 ) & $\mathrm{X}$ & & $\mathrm{X}$ \\
\hline Leptodactylus rhondomystax Boulenger, 1884 & $\mathrm{X}$ & $\mathrm{X}$ & $\mathrm{X}$ \\
\hline Leptodactylus stenodema Jiménez de la Espada, 1875 & & $\mathrm{X}$ & \\
\hline Lithodytes lineatus (Schneider, 1799) & $\mathrm{X}$ & $\mathrm{X}$ & $\mathrm{X}$ \\
\hline Engystomops petersi Jiménez de la Espada, 1872 & $\mathrm{X}$ & $\mathrm{X}$ & $\mathrm{X}$ \\
\hline Physalaemus ephippifer (Steindachner, 1864) & $\mathrm{X}$ & $\mathrm{X}$ & $\mathrm{X}$ \\
\hline Physalaemus sp. & & & $\mathrm{X}$ \\
\hline \multicolumn{4}{|l|}{ Family Strabomantidae } \\
\hline Pristimantis aff. ockendeni (Boulenger, 1912) & & $\mathrm{X}$ & $\mathrm{X}$ \\
\hline Pristimantis fenestratus (Steindachner, 1864) & $\mathrm{X}$ & $\mathrm{X}$ & $\mathrm{X}$ \\
\hline Pristimantis sp. & & $\mathrm{X}$ & $\mathrm{X}$ \\
\hline Pristimantis sp. 1 & & $\mathrm{X}$ & $\mathrm{X}$ \\
\hline Pristimantis sp. 2 & & $\mathrm{X}$ & $\mathrm{X}$ \\
\hline \multicolumn{4}{|l|}{ Family Hylidae } \\
\hline Dendropsophus brevifrons (Duellmann and Crump, 1974) & $\mathrm{X}$ & $\mathrm{X}$ & $\mathrm{X}$ \\
\hline Dendropsophus leucophyllatus (Beireis, 1783) & $\mathrm{X}$ & $\mathrm{X}$ & $\mathrm{X}$ \\
\hline Dendropsophus melanargyreus (Cope, 1887) & $\mathrm{X}$ & $\mathrm{X}$ & $\mathrm{X}$ \\
\hline Dendropsophus microcephalus (Cope, 1886) & & $\mathrm{X}$ & $\mathrm{X}$ \\
\hline Dendropsophus minutus (Peters, 1872) & & $\mathrm{X}$ & $\mathrm{X}$ \\
\hline Dendropsophus nanus (Boulenger, 1889) & & & $\mathrm{X}$ \\
\hline Dendropsophus sp. & & $\mathrm{X}$ & $\mathrm{X}$ \\
\hline Dendropsophus sp. 1 & $\mathrm{X}$ & & $\mathrm{X}$ \\
\hline Dendropsophus sp.2 & $\mathrm{X}$ & & $\mathrm{X}$ \\
\hline Dryaderces inframaculatus (Boulenger, 1882) & & $\mathrm{X}$ & $\mathrm{X}$ \\
\hline Hypsiboas albopunctatus (Spix, 1824) & & $\mathrm{X}$ & \\
\hline
\end{tabular}


Table 1. Continued...

\begin{tabular}{|c|c|c|c|}
\hline $\begin{array}{r}\text { Taxon } \\
\end{array}$ & EIA-RIMA & FRP & MEP \\
\hline Hypsiboas boans (Linnaeus, 1758) & $\mathrm{X}$ & $\mathrm{X}$ & $\mathrm{X}$ \\
\hline Hypsiboas calcaratus (Troschel, 1848) & $\mathrm{X}$ & $\mathrm{X}$ & $\mathrm{X}$ \\
\hline Hypsiboas cf. lanciformis (Cope, 1871) & & $\mathrm{X}$ & $\mathrm{X}$ \\
\hline Hypsiboas cinerascens (Spix, 1824) & $X$ & $\mathrm{X}$ & $\mathrm{X}$ \\
\hline Hypsiboas fasciatus (Günther, 1858) & $\mathrm{X}$ & & $\mathrm{X}$ \\
\hline Hypsiboas geographicus (Spix, 1824) & $\mathrm{X}$ & $X$ & $\mathrm{X}$ \\
\hline Hypsiboas multifasciatus (Günther, 1859) & $\mathrm{X}$ & $\mathrm{X}$ & $\mathrm{X}$ \\
\hline Hypsiboas raniceps Cope, 1862 & & $\mathrm{X}$ & $\mathrm{X}$ \\
\hline Hypsiboas sp. & & $\mathrm{X}$ & $\mathrm{X}$ \\
\hline Osteocephalus leprieurii (Duméril and Bibron, 1841) & $X$ & $\mathrm{X}$ & $\mathrm{X}$ \\
\hline Osteocephalus oophagus Jungfer and Schiesari, 1995 & $\mathrm{X}$ & $\mathrm{X}$ & \\
\hline Osteocephalus sp. & $\mathrm{X}$ & & \\
\hline Osteocephalus taurinus Steindachner, 1862 & $\mathrm{X}$ & $\mathrm{X}$ & $\mathrm{X}$ \\
\hline Phyllomedusa bicolor (Boddaert, 1772) & $\mathrm{X}$ & $\mathrm{X}$ & $\mathrm{X}$ \\
\hline Phyllomedusa hypochondrialis (Daudin, 1800) & $\mathrm{X}$ & $\mathrm{X}$ & $\mathrm{X}$ \\
\hline Phyllomedusa tomopterna (Cope, 1868$)$ & & $\mathrm{X}$ & $\mathrm{X}$ \\
\hline Phyllomedusa vaillantii Boulenger, 1882 & $\mathrm{X}$ & $\mathrm{X}$ & $\mathrm{X}$ \\
\hline Scinax aff. proboscideus (Brongersma, 1933) & & & $\mathrm{X}$ \\
\hline Scinax aff. rostratus (Peters, 1863) & & & $\mathrm{X}$ \\
\hline Scinax aff. $x$-signatus (Spix, 1824) & $\mathrm{X}$ & & \\
\hline Scinax boesemani (Goin, 1966) & $\mathrm{X}$ & $X$ & $\mathrm{X}$ \\
\hline Scinax cf. cruentommus (Duellman, 1972) & $\mathrm{X}$ & $\mathrm{X}$ & $\mathrm{X}$ \\
\hline Scinax fuscomarginatus (Lutz, 1925) & $\mathrm{X}$ & $\mathrm{X}$ & $\mathrm{X}$ \\
\hline Scinax garbei (Miranda-Ribeiro, 1926) & $\mathrm{X}$ & $\mathrm{X}$ & $\mathrm{X}$ \\
\hline Scinax nebulosus (Spix, 1824) & $\mathrm{X}$ & $\mathrm{X}$ & $\mathrm{X}$ \\
\hline Scinax rostratus (Peters, 1863) & & & $\mathrm{X}$ \\
\hline Scinax ruber (Laurenti, 1758) & $\mathrm{X}$ & & $\mathrm{X}$ \\
\hline Scinax sp. (S. ruber clade) & & & $\mathrm{X}$ \\
\hline Scinax sp.1 (S. ruber clade) & & & $\mathrm{X}$ \\
\hline Sphaenorhyncus lacteus (Daudin, 1800) & & & $\mathrm{X}$ \\
\hline Trachycephalus resifinictrix (Goeldi, 1907) & $\mathrm{X}$ & $\mathrm{X}$ & \\
\hline Trachycephalus typhonius (Linnaeus, 1758) & $\mathrm{X}$ & $\mathrm{X}$ & $\mathrm{X}$ \\
\hline \multicolumn{4}{|l|}{ Family Alophrynidae } \\
\hline Alophryne ruthveni Gaige, 1926 & $\mathrm{X}$ & $\mathrm{X}$ & $\mathrm{X}$ \\
\hline \multicolumn{4}{|l|}{ Family Centrolenidae } \\
\hline Cochranella adenocheira Harvey and Nooan, 2005 & & & $\mathrm{X}$ \\
\hline Cochranella sp. & & & $\mathrm{X}$ \\
\hline Hyalinobatrachium cappellei van Lidth de Jeude, 1904 & & & $\mathrm{X}$ \\
\hline Hyalinobatrachium iaspidiensis (Ayarzaqüena, 1992) & & $\mathrm{X}$ & \\
\hline Hyalinobatrachium sp. & $\mathrm{X}$ & $\mathrm{X}$ & $\mathrm{X}$ \\
\hline Hyalinobatrachium sp.1 & & & $\mathrm{X}$ \\
\hline Vitreorana ritae (Lutz, 1952) & & & $\mathrm{X}$ \\
\hline \multicolumn{4}{|l|}{ Family Aromobatidae } \\
\hline Allobates crombiei (Morales, 2002 “2000”) & $\mathrm{X}$ & $\mathrm{X}$ & $\mathrm{X}$ \\
\hline Allobates femoralis (Boulenger, 1884 “1883”) & $\mathrm{X}$ & $\mathrm{X}$ & $\mathrm{X}$ \\
\hline Allobates sp. & & $\mathrm{X}$ & $\mathrm{X}$ \\
\hline Allobates sp.1 & & & $\mathrm{X}$ \\
\hline Allobates sp. 2 & & & $\mathrm{X}$ \\
\hline \multicolumn{4}{|l|}{ Family Pipidae } \\
\hline Pipa arrabali Izecksohn, 1976 & & & $\mathrm{X}$ \\
\hline
\end{tabular}


Table 1. Continued...

\begin{tabular}{|c|c|c|c|}
\hline Taxon & EIA-RIMA & FRP & MEP \\
\hline Pipa pipa (Linnaeus, 1758) & & $\mathrm{X}$ & $\mathrm{X}$ \\
\hline \multicolumn{4}{|l|}{ Family Dendrobatidae } \\
\hline Adelphobates castaneoticus (Caldwell and Myers, 1990) & $\mathrm{X}$ & $\mathrm{X}$ & $\mathrm{X}$ \\
\hline Adelphobates galactonotus (Steindachner, 1864) & $\mathrm{X}$ & $\mathrm{X}$ & $\mathrm{X}$ \\
\hline Ameerega hahneli (Boulenger, 1884) & $\mathrm{X}$ & & $\mathrm{X}$ \\
\hline Ranitomeya amazonica (Schulte, 1999) & & & $\mathrm{X}$ \\
\hline \multicolumn{4}{|l|}{ Family Microhylidae } \\
\hline Chiasmocleis hudsoni Parker, 1940 & $\mathrm{X}$ & $\mathrm{X}$ & \\
\hline Chiasmocleis sp. & $\mathrm{X}$ & & \\
\hline Ctenophryne geayi Mocguard, 1904 & $\mathrm{X}$ & $\mathrm{X}$ & $\mathrm{X}$ \\
\hline Elachistocleis cf. carvalhoi Caramaschi, 2010 & $\mathrm{X}$ & $\mathrm{X}$ & $\mathrm{X}$ \\
\hline Hamptophryne boliviana (Parker, 1927) & $\mathrm{X}$ & & \\
\hline \multicolumn{4}{|l|}{ Order Urodela } \\
\hline \multicolumn{4}{|l|}{ Family Plethodontidae } \\
\hline Bolitoglossa paraensis (Unterstein, 1930) & & $\mathrm{X}$ & $\mathrm{X}$ \\
\hline Bolitoglossa tapajonica Brcko et al., 2013 & & $\mathrm{X}$ & \\
\hline \multicolumn{4}{|l|}{ Order Gymnophiona } \\
\hline \multicolumn{4}{|l|}{ Family Caecillidae } \\
\hline Caecilia gracilis Shaw, 1002 & & $\mathrm{X}$ & $\mathrm{X}$ \\
\hline Caecilia tentaculata Linnaeus, 1758 & & $\mathrm{X}$ & \\
\hline \multicolumn{4}{|l|}{ Family Siphonopidae } \\
\hline Microcaecilia $\mathrm{sp}$. & $\mathrm{X}$ & & \\
\hline Micricaecilia taylori Nussbaum and Hoogmoed, 1979 & & $\mathrm{X}$ & \\
\hline Siphonops annulatus (Mikan, 1820) & & & $X$ \\
\hline \multicolumn{4}{|l|}{ Family Typhlonectidae } \\
\hline Atretochoana eiselti (Taylor, 1968) & & $\mathrm{X}$ & \\
\hline Potomotyphlus kaupii (Berthold, 1859) & & $\mathrm{X}$ & \\
\hline Typhlonectes compressicauda (Duméril and Bibron, 1841) & & $\mathrm{X}$ & \\
\hline
\end{tabular}

\section{Class Reptilia}

\section{Order Squamata}

\section{Suborder Amphisbaenia}

\section{Family Amphisbaenidae}

Amphisbaena alba Linnaeus, 1758

Amphisbaena amazonica Vanzolini, 1951

Amphisbaena brasiliana (Gray, 1865)

Amphisbaena mitchelli Procter, 1923

\section{Suborder Sauria}

\section{Family Gekkonidae}

Hemidactylus mabouia (Moreau de Jonnès, 1818)

Family Phyllodactylidae

\begin{tabular}{|c|c|c|c|}
\hline Thecadactylus rapicauda (Houttuyn, 1782) & $\mathrm{X}$ & $\mathrm{X}$ & $\mathrm{X}$ \\
\hline \multicolumn{4}{|l|}{ Family Sphaerodactylidae } \\
\hline Chatogeckko amazonicus (Andersson, 1918) & $\mathrm{X}$ & $\mathrm{X}$ & $\mathrm{X}$ \\
\hline Gonatodes hasemani Griffin, 1917 & $\mathrm{X}$ & $\mathrm{X}$ & $\mathrm{X}$ \\
\hline Gonatodes humeralis (Guichenot, 1855) & $\mathrm{X}$ & $\mathrm{X}$ & $\mathrm{X}$ \\
\hline Gonatodes nascimentoi Sturaro and Ávila-Pires, 2011 & & & $\mathrm{X}$ \\
\hline Gonatodes eladioi Nascimento, Ávila-Pires and Cunha, 1987 & & $\mathrm{X}$ & \\
\hline Lepidoblepharis heyerorum Vanzolini, 1978 & $\mathrm{X}$ & $\mathrm{X}$ & $\mathrm{X}$ \\
\hline Pseudogonatodes guianensis Parker, 1935 & $\mathrm{X}$ & $\mathrm{X}$ & $\mathrm{X}$ \\
\hline
\end{tabular}

Family Gymnophthalmidae 
Table 1. Continued...

\begin{tabular}{|c|c|c|c|}
\hline Taxon & EIA-RIMA & FRP & MEP \\
\hline Alopoglossus angulatus (Linnaeus, 1758) & $\mathrm{X}$ & $\mathrm{X}$ & $\mathrm{X}$ \\
\hline Arthrosaura kockii (Lidth de Jeude, 1904) & $\mathrm{X}$ & & $\mathrm{X}$ \\
\hline Arthrosaura reticulata (O'Shaughnessy, 1881) & $\mathrm{X}$ & $\mathrm{X}$ & $\mathrm{X}$ \\
\hline Colobosaura modesta (Reinhardt and Luetken, 1862) & & $\mathrm{X}$ & \\
\hline Cercosaura argulus Peters, 1863 & $\mathrm{X}$ & $\mathrm{X}$ & \\
\hline Cercosaura ocellata Wagler, 1830 & $\mathrm{X}$ & $\mathrm{X}$ & $\mathrm{X}$ \\
\hline Leposama osvaldoi Ávila-Pires, 1995 & & $\mathrm{X}$ & $\mathrm{X}$ \\
\hline Leposoma percarinatum (Müller, 1923) & $\mathrm{X}$ & $\mathrm{X}$ & $\mathrm{X}$ \\
\hline Leposoma sp. & & & $\mathrm{X}$ \\
\hline Leposoma sp.1 & & & $\mathrm{X}$ \\
\hline Neusticurus rudis Boulenger, 1900 & & & $\mathrm{X}$ \\
\hline Potamipes ecpleopus (Cope, 1876$)$ & & $\mathrm{X}$ & $\mathrm{X}$ \\
\hline Ptychoglossus brevifrontalis Boulenger, 1912 & $\mathrm{X}$ & $\mathrm{X}$ & \\
\hline Tretioscincus agilis Ruthven, 1916 & $\mathrm{X}$ & & $\mathrm{X}$ \\
\hline \multicolumn{4}{|l|}{ Family Iguanidae } \\
\hline Iguana iguana (Linnaeus, 1758) & $\mathrm{X}$ & $\mathrm{X}$ & $\mathrm{X}$ \\
\hline \multicolumn{4}{|l|}{ Family Dactyloidae } \\
\hline Anolis fuscoauratus D’Orbigny, 1837 & $\mathrm{X}$ & $\mathrm{X}$ & $\mathrm{X}$ \\
\hline Anolis ortonii Cope, 1868 & $\mathrm{X}$ & $\mathrm{X}$ & $\mathrm{X}$ \\
\hline Anolis punctatus Daudin, 1802 & $\mathrm{X}$ & $\mathrm{X}$ & \\
\hline Anolis sp. & & $\mathrm{X}$ & $\mathrm{X}$ \\
\hline Anolis trachyderma Cope, 1876 & $\mathrm{X}$ & $\mathrm{X}$ & $\mathrm{X}$ \\
\hline \multicolumn{4}{|l|}{ Fmily Leiosauridae } \\
\hline Enyalius leechi (Boulenger, 1885) & & $\mathrm{X}$ & $\mathrm{X}$ \\
\hline \multicolumn{4}{|l|}{ Family Polychrotidae } \\
\hline Polychrus marmoratus (Linnaeus, 1758) & $\mathrm{X}$ & $\mathrm{X}$ & $\mathrm{X}$ \\
\hline \multicolumn{4}{|l|}{ Family Scincidae } \\
\hline Copeoglossum nigropunctatum (Spix, 1825) & $\mathrm{X}$ & $\mathrm{X}$ & $\mathrm{X}$ \\
\hline \multicolumn{4}{|l|}{ Family Tropiduridae } \\
\hline Plica plica (Linnaeus, 1758) & $\mathrm{X}$ & $\mathrm{X}$ & $\mathrm{X}$ \\
\hline Plica umbra (Linnaeus, 1758) & $\mathrm{X}$ & $\mathrm{X}$ & $\mathrm{X}$ \\
\hline Uranocentron azureum (Linnaeus, 1758) & & $\mathrm{X}$ & \\
\hline Uranoscondon superciliosus (Linnaeus, 1758) & $\mathrm{X}$ & $\mathrm{X}$ & $\mathrm{X}$ \\
\hline \multicolumn{4}{|l|}{ Family Teiidae } \\
\hline Ameiva ameiva (Linnaeus, 1758) & $\mathrm{X}$ & $\mathrm{X}$ & $\mathrm{X}$ \\
\hline Cnemidophorus cryptus Cole and Dessauer, 1993 & $\mathrm{X}$ & $\mathrm{X}$ & $\mathrm{X}$ \\
\hline Crocodilurus amazonicus Spix, 1825 & & & $\mathrm{X}$ \\
\hline Kentropys calcarata Spix, 1825 & $\mathrm{X}$ & $\mathrm{X}$ & $\mathrm{X}$ \\
\hline Kentropyx altamazonica (Cope, 1876) & & $\mathrm{X}$ & $\mathrm{X}$ \\
\hline Tupinambis teguixin (Linnaeus, 1758) & $\mathrm{X}$ & $\mathrm{X}$ & $\mathrm{X}$ \\
\hline \multicolumn{4}{|l|}{ Subordem Serpentes } \\
\hline \multicolumn{4}{|l|}{ Family Aniliidae } \\
\hline Anilius scytale (Linnaeus, 1758) & & $\mathrm{X}$ & $\mathrm{X}$ \\
\hline \multicolumn{4}{|l|}{ Family Anomalepididae } \\
\hline Liotyphlops ternetzi (Boulenger, 1896) & & $\mathrm{X}$ & \\
\hline \multicolumn{4}{|l|}{ Family Boidae } \\
\hline Boa constrictor (Linnaeus, 1758) & $\mathrm{X}$ & $\mathrm{X}$ & $\mathrm{X}$ \\
\hline Corallus caninus (Linnaeus, 1758) & $\mathrm{X}$ & $\mathrm{X}$ & \\
\hline Corallus hortulanus (Linnaeus, 1758) & $\mathrm{X}$ & $\mathrm{X}$ & $\mathrm{X}$ \\
\hline Epicrates cenchria (Linnaeus, 1758) & $\mathrm{X}$ & $\mathrm{X}$ & $\mathrm{X}$ \\
\hline
\end{tabular}


Table 1. Continued...

\begin{tabular}{|c|c|c|c|}
\hline Taxon & EIA-RIMA & FRP & MEP \\
\hline Eunectes murinus (Linnaeus, 1758) & $\mathrm{X}$ & $\mathrm{X}$ & \\
\hline \multicolumn{4}{|l|}{ Family Colubridae } \\
\hline Apostolepis nigrolineata (Peters, 1896) & & $\mathrm{X}$ & $\mathrm{X}$ \\
\hline Apostolepis pymi Boulenger, 1903 & & $\mathrm{X}$ & \\
\hline Atractus albuquerquei Cunha and Nascimento, 1983 & $X$ & $\mathrm{X}$ & \\
\hline Atractus elaps (Günther, 1858) & & $\mathrm{X}$ & \\
\hline Atractus latifrons (Günther, 1868) & & $\mathrm{X}$ & \\
\hline Atractus schach (Boie, 1827) & $\mathrm{X}$ & $\mathrm{X}$ & \\
\hline Atractus snethlageae Cunha and Nascimento, 1983 & $\mathrm{X}$ & $\mathrm{X}$ & $\mathrm{X}$ \\
\hline Atractus sp. & & $\mathrm{X}$ & \\
\hline Chironius bicarinatus (Wied, 1820) & & $\mathrm{X}$ & \\
\hline Chironius carinatus (Linnaeus, 1758) & & $\mathrm{X}$ & $\mathrm{X}$ \\
\hline Chironius exoletus (Linnaeus, 1758) & & $\mathrm{X}$ & $\mathrm{X}$ \\
\hline Chironius fuscus (Linnaeus, 1758) & $\mathrm{X}$ & $\mathrm{X}$ & $\mathrm{X}$ \\
\hline Chironius multiventris Schmidt and Walker, 1943 & $\mathrm{X}$ & $\mathrm{X}$ & $\mathrm{X}$ \\
\hline Chironius scurrulus (Wagler, 1824) & $\mathrm{X}$ & $\mathrm{X}$ & $\mathrm{X}$ \\
\hline Clelia cleia (Daudin, 1803) & $\mathrm{X}$ & $\mathrm{X}$ & \\
\hline Clelia plumbea (Wied, 1820) & & & $\mathrm{X}$ \\
\hline Dendrophidion dendrophis (Schlegel, 1837) & & $\mathrm{X}$ & $\mathrm{X}$ \\
\hline Dipsas catesbyi (Sentzen, 1796) & $\mathrm{X}$ & $\mathrm{X}$ & $X$ \\
\hline Dipsas indica Laurenti, 1768 & & $\mathrm{X}$ & $\mathrm{X}$ \\
\hline Dipsas pavonina Schlegel, 1837 & & $\mathrm{X}$ & \\
\hline Dipsas variegata (Duméril, Bibron and Duméril, 1854) & & $\mathrm{X}$ & \\
\hline Drepanoides anomalus (Jan, 1863) & $\mathrm{X}$ & $\mathrm{X}$ & $\mathrm{X}$ \\
\hline Drymarchon corais (Boie, 1827) & & $\mathrm{X}$ & \\
\hline Drymoluber dichrous (Peters, 1863) & & $\mathrm{X}$ & $\mathrm{X}$ \\
\hline Erythrolamprus aesculapii (Linnaeus, 1766) & & $\mathrm{X}$ & \\
\hline Erythrolamprus breviceps (Cope, 1860) & & $\mathrm{X}$ & \\
\hline Erythrolamprus oligolepis (Boulenger, 1905) & $\mathrm{X}$ & & \\
\hline Erythrolamprus poecilogyrus (Wied, 1825) & & $\mathrm{X}$ & \\
\hline Erythrolamprus reginae (Linnaeus, 1758) & $\mathrm{X}$ & & $X$ \\
\hline Erythrolamprus taeniogaster (Jan, 1863) & & $\mathrm{X}$ & \\
\hline Erythrolamprus typhlus (Linnaeus, 1758) & $\mathrm{X}$ & $\mathrm{X}$ & \\
\hline Helicops angulatus (Linnaeus, 1758) & $\mathrm{X}$ & $\mathrm{X}$ & $\mathrm{X}$ \\
\hline Helicops modestus Günther, 1861 & & $\mathrm{X}$ & \\
\hline Hydrodynastes bicinctus (Herrmann, 1804) & $\mathrm{X}$ & $\mathrm{X}$ & \\
\hline Hydrodynastes gigas (Duméril, Bibron and Duméril, 1854) & & $\mathrm{X}$ & \\
\hline Hydrops martii (Wagler, 1824) & $\mathrm{X}$ & $\mathrm{X}$ & \\
\hline Hydrops triangularis (Wagler, 1824) & & $\mathrm{X}$ & \\
\hline Imantodes cenchoa (Linnaeus, 1758) & $\mathrm{X}$ & $\mathrm{X}$ & $\mathrm{X}$ \\
\hline Imantodes lentiferus (Cope, 1894) & & $\mathrm{X}$ & \\
\hline Leptodeira annulata (Linnaeus, 1758) & $\mathrm{X}$ & $\mathrm{X}$ & $\mathrm{X}$ \\
\hline Leptophis ahaetulla (Linnaeus, 1758) & $\mathrm{X}$ & $\mathrm{X}$ & $\mathrm{X}$ \\
\hline Mastigodryas boddaerti (Sentzen, 1796) & $\mathrm{X}$ & $\mathrm{X}$ & $\mathrm{X}$ \\
\hline Oxybelis aeneus (Wagler, 1824) & $\mathrm{X}$ & $\mathrm{X}$ & $\mathrm{X}$ \\
\hline Oxybelis fulgidus (Daudin, 1803) & & $\mathrm{X}$ & $\mathrm{X}$ \\
\hline Oxyrhopus melanogenys (Tschudi, 1845) & $\mathrm{X}$ & $\mathrm{X}$ & $\mathrm{X}$ \\
\hline Oxyrhopus petolarius Reuss, 1834 & & $\mathrm{X}$ & $\mathrm{X}$ \\
\hline Oxyrhopus trigeminus Duméril, Bibron and Duméril, 1854 & $\mathrm{X}$ & $\mathrm{X}$ & $\mathrm{X}$ \\
\hline Philodryas argentea (Daudin, 1803) & $\mathrm{X}$ & & $\mathrm{X}$ \\
\hline
\end{tabular}


Table 1. Continued...

\begin{tabular}{|c|c|c|c|}
\hline Taxon & EIA-RIMA & FRP & MEP \\
\hline Philodryas olfersii (Lichtenstein, 1823) & & $\mathrm{X}$ & \\
\hline Philodryas viridissima (Linnaeus, 1758) & $\mathrm{X}$ & $\mathrm{X}$ & \\
\hline Pseudoboa coronata Schneider, 1801 & $\mathrm{X}$ & $\mathrm{X}$ & $\mathrm{X}$ \\
\hline Pseudoboa nigra (Duméril, Bibron and Duméril, 1854) & & $\mathrm{X}$ & \\
\hline Pseudoeryx plicatilis (Linnaeus, 1758) & & $\mathrm{X}$ & \\
\hline Pseustes sulphureus (Wagler, 1824) & $\mathrm{X}$ & $\mathrm{X}$ & \\
\hline Pseutes poecilonotus (Peters, 1867) & & $\mathrm{X}$ & \\
\hline Rhinobotrium lentiginosum (Scopoli, 1785) & & $\mathrm{X}$ & $\mathrm{X}$ \\
\hline Sibon nebulatus (Linnaeus, 1758) & $\mathrm{X}$ & $\mathrm{X}$ & $\mathrm{X}$ \\
\hline Siphlophis cervinus (Laurenti, 1768) & $\mathrm{X}$ & $\mathrm{X}$ & $\mathrm{X}$ \\
\hline Siphlophis compressus (Daudin, 1803) & $\mathrm{X}$ & $\mathrm{X}$ & $\mathrm{X}$ \\
\hline Siphlophis worontzowi (Prado, 1940) & & $\mathrm{X}$ & \\
\hline Spilotes pullatus (Linnaeus, 1758) & $\mathrm{X}$ & $\mathrm{X}$ & $\mathrm{X}$ \\
\hline Taeniophallus brevirostris (Peters, 1863) & $\mathrm{X}$ & $\mathrm{X}$ & \\
\hline Taeniophallus quadriocelatus Santos-Jr., Di-Bernardo and Lema, 2008 & & $\mathrm{X}$ & $\mathrm{X}$ \\
\hline Tantilla melanocephala (Linnaeus, 1758) & $\mathrm{X}$ & $\mathrm{X}$ & $\mathrm{X}$ \\
\hline Xenodon merremi (Wagler, 1824) & & $\mathrm{X}$ & \\
\hline Xenodon rabdocephalus (Wied, 1824) & $\mathrm{X}$ & $\mathrm{X}$ & \\
\hline Xenodon severus (Linnaeus, 1758) & $\mathrm{X}$ & $\mathrm{X}$ & $\mathrm{X}$ \\
\hline Xenopholis scalaris (Wucherer, 1861) & $\mathrm{X}$ & $\mathrm{X}$ & $\mathrm{X}$ \\
\hline \multicolumn{4}{|l|}{ Family Elapidae } \\
\hline Micrurus filiformis (Günther, 1859) & & $\mathrm{X}$ & \\
\hline Micrurus hemprichii (Jan, 1858) & & $\mathrm{X}$ & $\mathrm{X}$ \\
\hline Micrurus lemniscatus (Linnaeus, 1758) & $\mathrm{X}$ & $\mathrm{X}$ & $\mathrm{X}$ \\
\hline Micrurus paraensis Cunha and Nascimento, 1973 & & $\mathrm{X}$ & $\mathrm{X}$ \\
\hline Micrurus psyches (Daudin, 1803) & & $\mathrm{X}$ & \\
\hline Micrurus spixii Wagler, 1824 & $\mathrm{X}$ & $\mathrm{X}$ & \\
\hline Micrurus surinamensis (Cuvier, 1817) & & $\mathrm{X}$ & $\mathrm{X}$ \\
\hline \multicolumn{4}{|l|}{ Family Typhlopidae } \\
\hline Typhlops brongersmianus Vanzolini, 1976 & & $\mathrm{X}$ & \\
\hline Typhlops reticulatus (Linnaeus, 1758) & $\mathrm{X}$ & $\mathrm{X}$ & $\mathrm{X}$ \\
\hline \multicolumn{4}{|l|}{ Family Leptotyphlopidae } \\
\hline Siagonodon septemstriatus (Schneider, 1801) & & $\mathrm{X}$ & \\
\hline Trilepida macroleps (Peters, 1857) & & $\mathrm{X}$ & $\mathrm{X}$ \\
\hline \multicolumn{4}{|l|}{ Family Viperidae } \\
\hline Bothrops bilineata (Wied, 1825) & & $\mathrm{X}$ & \\
\hline Bothrops taeniata (Wagner, 1824) & & $\mathrm{X}$ & \\
\hline Bothrops atrox (Linnaeus, 1758) & $\mathrm{X}$ & $\mathrm{X}$ & $\mathrm{X}$ \\
\hline Lachesis muta (Linnaeus, 1766) & $\mathrm{X}$ & $\mathrm{X}$ & $\mathrm{X}$ \\
\hline \multicolumn{4}{|l|}{ Order Crocodylia } \\
\hline \multicolumn{4}{|l|}{ Family Alligatoridae } \\
\hline Caiman crocodilus (Linnaeus, 1758) & $X$ & $\mathrm{X}$ & $\mathrm{X}$ \\
\hline Melanosuchus niger (Spix, 1825) & $\mathrm{X}$ & & \\
\hline Paleosuchus palpebrosus (Cuvier, 1807) & $\mathrm{X}$ & & $\mathrm{X}$ \\
\hline Paleosuchus trigonatus (Schneider, 1801) & $\mathrm{X}$ & $\mathrm{X}$ & $\mathrm{X}$ \\
\hline \multicolumn{4}{|l|}{ Order Testudines } \\
\hline \multicolumn{4}{|l|}{ Family Chelidae } \\
\hline Mesoclemmys gibba (Schweigger, 1812) & $\mathrm{X}$ & $\mathrm{X}$ & $\mathrm{X}$ \\
\hline Platemys platycephala (Schneider, 1792) & $\mathrm{X}$ & $\mathrm{X}$ & $\mathrm{X}$ \\
\hline
\end{tabular}


Table 1. Continued...

\begin{tabular}{|c|c|c|c|}
\hline Taxon & EIA-RIMA & FRP & MEP \\
\hline \multicolumn{4}{|l|}{ Family Geoemydidae } \\
\hline Rhinoclemmys punctularia (Daudin, 1801) & $\mathrm{X}$ & $\mathrm{X}$ & $\mathrm{X}$ \\
\hline \multicolumn{4}{|l|}{ Family Knosternidae } \\
\hline Kinosternon scorpioidis (Linnaeus, 1766) & $\mathrm{X}$ & $\mathrm{X}$ & \\
\hline \multicolumn{4}{|l|}{ Family Podocnemididae } \\
\hline Peltocephalus dumerilianus (Schweigger, 1812) & $\mathrm{X}$ & & \\
\hline Podocnemis expansa (Schweigger, 1812) & $\mathrm{X}$ & $\mathrm{X}$ & \\
\hline Podocnemis sextuberculata Cornalia, 1849 & $\mathrm{X}$ & & \\
\hline Podocnemis unifilis Troschel, 1848 & $\mathrm{X}$ & $\mathrm{X}$ & \\
\hline \multicolumn{4}{|l|}{ Family Testudinidae } \\
\hline Chelonoidis carbonarius (Spix, 1824) & $\mathrm{X}$ & & $\mathrm{X}$ \\
\hline Chelonoidis denticulatus (Linnaeus, 1766) & $\mathrm{X}$ & & $\mathrm{X}$ \\
\hline
\end{tabular}

studies discuss aspects of reproductive behaviour and how sympatric species are ecologically segregated (Rojas-Morales and Escobar-Lasso, 2013). The genus Pristimantis and Allobates show a high cryptic diversity and other genus reveal the presence of taxonomic complex (for example, Adenomera, Dendropsophus, Scinax, Osteocephalus, and Leposoma). Some semi-fossorial species represent undescribed species, for example, for Atractus (P. Passos, pers. com.) and Proceratophrys (W. Vaz-Silva, pers. obs.). The record of rare gymnophiona Atretochoana eiselti reveals the biogeographic importance in the regional context.

The diversity profiles of amphibian assemblages (Figure 2) show that M5 present the highest richness with 68 species $(a=0)$, while M1 and M2 the poorest, with 45 and 46 species respectively. Considering the diversity, the amphibian assemblages are very similar, and as pointed out by Tóthmérész (1995) and Melo (2008), it is not possible to distinguish them using Hill's Series Diversity Profile. The high values of $a$ parameter obtained by Hill's Profile give the evenness of the assemblages. Therefore, M2 and M8 present the less uniform species proportions, while the highest evenness was observed in M1 and M5.

Regarding reptile diversity, the assemblages are very similar. The highest richness is found in M6 with 50 species (Figure 3). On the other hand, M3 and M4 presented the least number of registered species, 33 . The diversity profiles show that the less uniform assemblages are present in M3 and M4. The reptile assemblages are more susceptible to a low evenness index, giving the cryptic habitats of many species, which make them barely detectable, resulting in occasional registers.

According to Melo (2008), understanding the diversity of species in an area is fundamental for optimising management in relation to the activity of exploitation of low impact, resource conservation or for the restoration of degraded ecosystems. Despite showing similar phytophysiognomies, the modules are under the influence of several factors (e.g. the edge effect), due to the size and disposition of remains in the landscape. Edge and area effects are the most important factors that cause changes in fragmented communities (Nascimento and Laurance, 2006). Results of the diversity in the modules may be related to habitat characteristics (humidity, temperature, incorporation of litter, litter depth and changes in vegetation structure edge or lower strata of the forest margin) with the consequent availability of resources, as well as reflecting patterns of spatial distribution of species.

The cluster analysis of the amphibian assemblages generated two groups (Figure 4). The module M1 showed a more differentiated assemblage. Modules M5, M6 and M7 are located on the left bank of the Xingu River, and presented high values of abundance and richness. The results can be attributed to the similar characteristics between the sites. The other grouping is the modules on the right bank of the Xingu River, in addition to M2.

In relation to the reptile assemblage, the cluster analysis formed two clusters that are represented by modules present on opposite banks of the Xingu River (Figure 5). Reptile assemblages can be differentiated by the environmental attributes on each bank, or the river can be a barrier to the species. The similarity between the two banks is less between themselves than modules on the same bank. According to biogeographic hypothesis, rivers may play a major role in creating and maintaining high levels of species diversity in the tropics increasing the possibility of allopatric speciation (Gascon et al., 1998).

The regional species richness can still be considered underestimated, considering the taxonomic uncertainty, complexity and cryptic diversity of various species, as observed in other regions of the Amazon biome (see Padial and De La Riva, 2009; Funk et al., 2011; Jansen et al., 2011). Considering other experiences observed within hydroelectric projects, the impacts of biodiversity are irreversible mainly due to forest loss, leading to the loss of natural ecosystems (Fearnside, 2001). Our results represent the actual regional knowledge before the implementation of the UHE Belo Monte, and they are important for permitting future comparison after the operation of the power plant. Considering the high diversity of the region and the predicted impacts, we suggest that scientific collection should be prioritised for the production of scientific knowledge. Studies related to integrative taxonomy are 

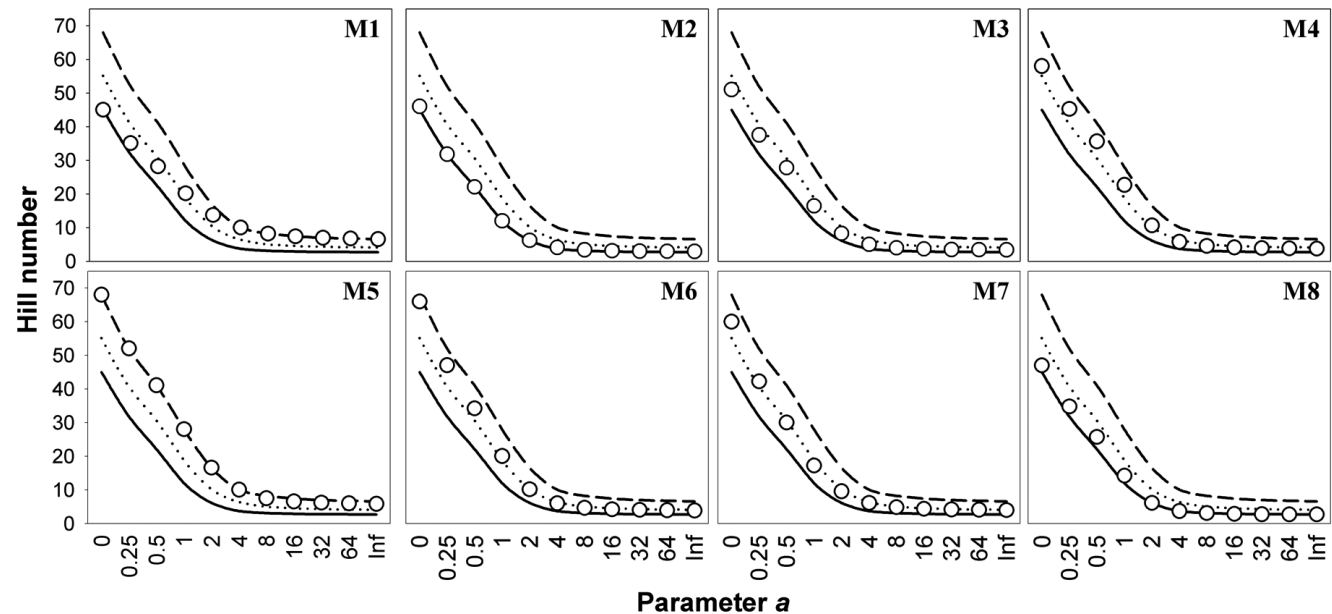

Figure 2. Profiles of assemblage diversity (for amphibians) in RAPELD modules. The three lines represent the maximum, average and minimum diversity values considering all modules. Empty circles represent the value of the Hill number for the module. M1 - M8 = Modules 1 to 8 .
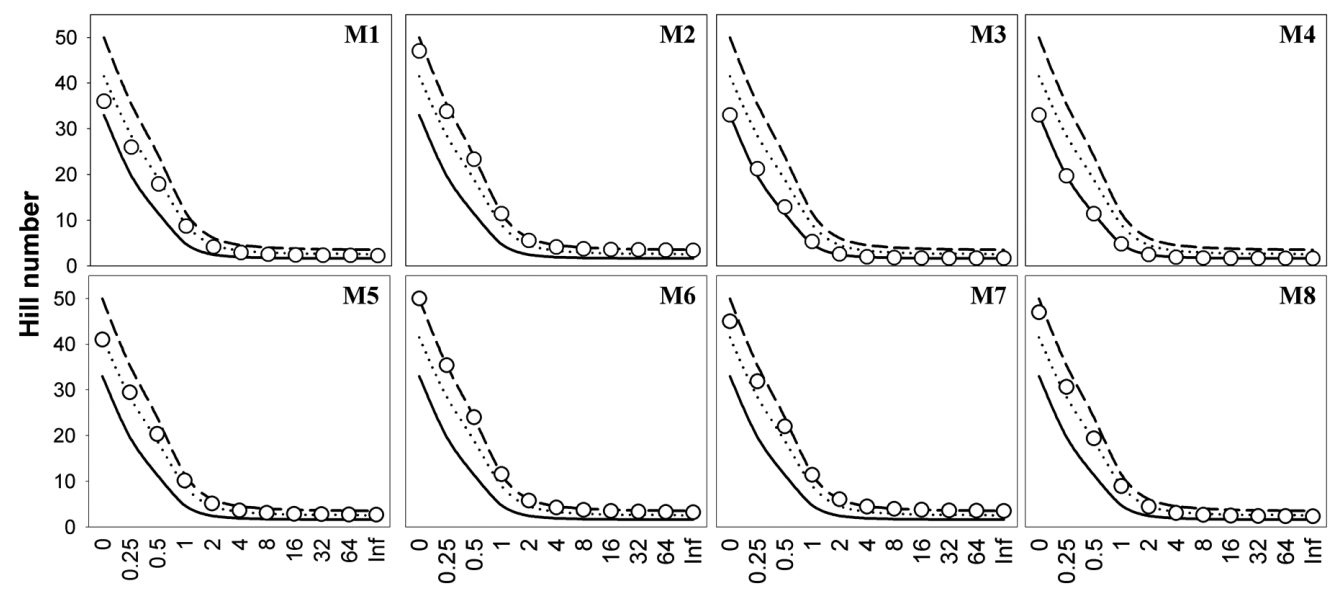

Parameter $a$

Figure 3. Profiles of assemblage diversity (for reptiles) in RAPELD modules. The three lines represent the maximum, average and minimum values diversity considering all modules. Empty circles represent the value of the Hill number for the module. M1 - M8 = Modules 1 to 8 .

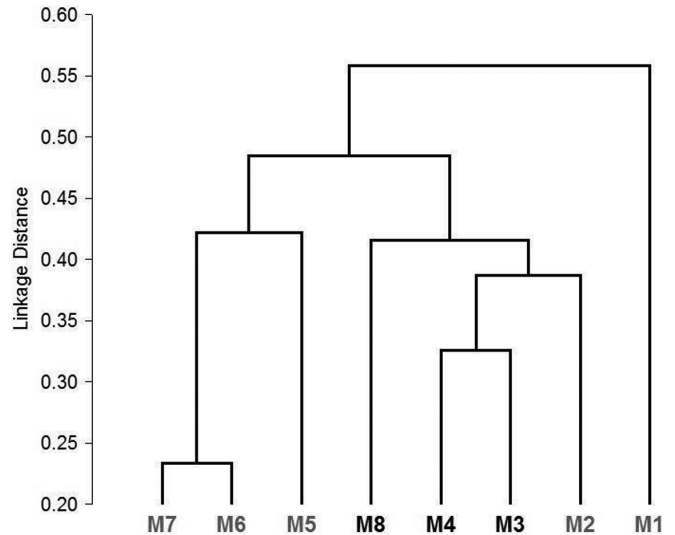

Figure 4. Cluster analyses of the amphibian assemblages in RAPELD modules. Modules in bold (M3, M4, M8) are located on the right bank of the Xingu river, and the others (M1, M2, M5, M6, M7) are located on the left bank.

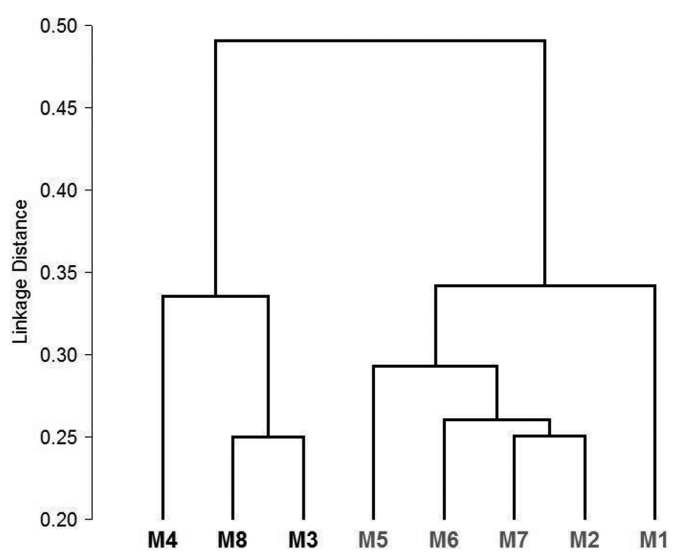

Figure 5. Cluster analyses of the reptiles assemblages in RAPELD modules. Modules in bold (M3, M4, M8) is located in the right bank of the Xingu river, and others (M1, M2, M5, M6, M7) is located in the left bank. 
needed to elucidate the uncertainties and increase levels of local diversity.

\section{Acknowledgements}

We thank the Biota Projetos e Consultoria Ambiental, Leme Engenharia and Norte Energia for logistical and financial support. Rafael C. Teixeira, Julimar A. Oliveira, and Samuel F. dos Anjos for assistance in the fieldwork. Ronaldo Carneiro, Carla Alves, Pablo V. C. Mathias, Cláudio V. Mendonça, and Joyce Costa for institutional support and assistance in the execution of Environmental Programmes. Sean Michael Quail for contributions to the manuscript. The programmes are conducted under IBAMA license numbers 251/2013 and 108/2012.

\section{References}

ÁVILA-PIRES, TCS., HOOGMOED, MS. and VITT, LJ., 2007. Herpetofauna da Amazônia. In NASCIMENTO, LB. and OLIVEIRA, ME. (Eds.). Herpetologia no Brasil II. Belo Horizonte: Sociedade Brasileira de Herpetologia. p. 13-43.

AVILA-PIRES, TCS., VITT, LJ., SARTORIUS, SS., and ZANI, PA., 2009. Squamata (Reptilia) from four sites in southern Amazonia, with a biogeographic analysis of Amazonian lizards. Boletim do Museu Paraense Emílio Goeldi. Ciências Naturais, vol. 4, no. 2, p. 99-118.

AZEVEDO-RAMOS, C. and GALATTI, U., 2002. Patterns of amphibian diversity in Brazilian Amazonia: conservation implications. Biological Conservation, vol. 103, no. 1, p. 103-111. http://dx.doi.org/10.1016/S0006-3207(01)00129-X.

BERNARDE, PS. and ABE, AS., 2006. A snake community at Espigão do Oeste, Rondônia, Southwestern Amazon, Brazil. South American Journal of Herpetology, vol. 1, no. 2, p. 102-113. http:// dx.doi.org/10.2994/1808-9798(2006)1[102:ASCAED]2.0.CO;2.

BERNARDE, PS. and MACEDO, LC., 2008. Impacto do desmatamento e formação de pastagens sobre a anurofauna de serapilheira em Rondônia. Iheringia. Zoologia, vol. 98, no. 4, p. 454-459. http://dx.doi.org/10.1590/S0073-47212008000400006.

BERNARDE, PS., 2007. Ambientes e temporada de vocalização da anurofauna no Município de Espigão do Oeste, Rondônia, Sudoeste da Amazônia - Brasil (Amphibia: Anura). Biota Neotropica, vol. 7, no. 2, p. 87-92. http://dx.doi.org/10.1590/ S1676-06032007000200010.

BERNARDE, PS., MACHADO, RA. and TURCI, LCB., 2011. Herpetofauna da área do Igarapé Esperança da Reserva Extrativista Riozinho da Liberdade, Acre, Brasil. Biota Neotropica, vol. 11, no. 3, p. 117-144. http://dx.doi.org/10.1590/S1676-06032011000300010.

BITAR, YOC., PINHEIRO, LPC., STURARO, MJ., FROTA, JG., COSTA, MCS and PRUDENTE, AL., 2011. Anuros do médio Rio Tapajós, oeste do Pará, Brasil: novos registros para a região. In Anais do IX Congresso Latino Americano de Herpetologia e V Congresso Brasileiro de Herpetologia, 2011. Curitiba. Curitiba: Sociedade Brasileira de Herpetologia.

BRCKO, IS., HOOGMOED, MS. and NECKEL-OLIVEIRA, S., 2013. Taxonomy and distribution of the salamander genus Bolitoglossa Duméril, Bibron \& Duméril, 1854 (Amphibia, Caudata, Plethodontidae) in Brazilian Amazonia. Zootaxa, vol. 3686, no. 4, p. 401-431. http://dx.doi.org/10.11646/zootaxa.3686.4.1.
CALDWELL, JP. and ARAUJO, MC., 2005. Amphibian faunas of two eastern Amazonian rainforest sites in Pará, Brazil. Occasional Papers Sam Noble Oklahoma Museum of Natural History, vol. 16, p. 1-41.

CALDWELL, JP. and LOPEZ, PT., 1989. Foam generating behavior in tadpoles of Leptodactylus mystaceus. Copeia, vol. 1989, no. 2, p. 498-502. http://dx.doi.org/10.2307/1445453.

CAMERA, BF. and KRINSKI, D., 2014. Distribution extension and geographic distribution map of the Brazil-nut poison dark frog Adelphobates castaneoticus (Caldwell \& Myers, 1990) (Anura: Dendrobatidae): new record for southwestern Pará State, Brazil. Checklist, vol. 10, no. 1, p. 244-245.

CISNEROS-HEREDIA, DF. and MCDIARMID, RW., 2007. Revision of the characters of Centrolenidae (Amphibia: Anura: Athespha- tanura), with comments on its taxonomy and the description of new taxa of glassfrogs. Zootaxa, vol. 1572, p. 1-82.

CISNEROS-HEREDIA, DF., STRUSSMANN, C., AVILA, RW., KAWASHITA-RIBEIRO, RA., 2010. Amphibia, Anura, Centrolenidae, Hyalinobatrachium carlesvilai Castroviejo-Fisher, Padial, Chaparro, Aguayo and de la Riva, 2009: first country record, Brazil. Check List, vol. 6, no. 2, p. 225-226.

COE, MT., MARTHEWS, TR., COSTA, MH., GALBRAITH, DR., GREENGLASS, NL., IMBUZEIRO, HMA., LEVINE, NM., MALHI, Y., MOORCROFT, PR., MUZA, MN., POWELL, TL., SALESKA, SR., SOLORZANO, LA. and WANG, J., 2013. Deforestation and climate feedbacks threaten the ecological integrity of south-southeastern Amazonia. Philosophical transactions of the Royal Society of London. Series B, Biological Sciences, vol. 368, no. 1619 , p. 20120155. http://dx.doi.org/10.1098/rstb.2012.0155. PMid:23610166.

FEARNSIDE, PM., 2001. Environmental impacts of Brazil's Tucuruí Dam: unlearned lessons for hydroelectric development in Amazonia. Environmental Management, vol. 27, no. 3, p. 377-396. http://dx.doi.org/10.1007/s002670010156. PMid:11148764.

FERRÃO, M., RODRIGUES-FILHO, JAS. and SILVA, M., 2012. O. Checklist of reptiles (Testudines, Squamata) from Alto Alegre dos Parecis, southweastern Amazonia, Brazil. Herpetology Notes, vol. 5, p. 473-480.

FRANÇA, FGR. and VENÂNCIO, NM., 2010. Reptiles and amphibians of a poorly known region in southwest Amazonia. Biotemas, vol. 23, no. 3, p. 71-84.

FROST, DR., 2014. Amphibian species of the world: an online reference. American Museum of Natural History. Version 6.0. Available from: $<$ http://research.amnh.org/herpetology/amphibia/ index.html>. Access in: 20 May 2014.

FROTA, JG. and VAZ-SILVA, W., 2013. Dendropsophus sarayacuensis (Shreve, 1935) (Amphibia, Anura): filling gap on the geographic distribution. Check List, vol. 9, no. 1, p. 129-130. http://dx.doi.org/10.15560/9.1.129.

FROTA, JG., 2004. As serpentes da região de Itaituba, médio Rio Tapajós, Pará, Brasil (Squamata). Comunicações do Museu de Ciências e Tecnologia da PUCRS, vol. 17, no. 1. p. 9-19.

FROTA, JG., PRUDENTE, ALC., D'ANGIOLELLA, AB., SANTOS-COSTA, MC and FERREIRA, A., 2011. Serpentes do Parque Nacional (PARNA) da Amazônia, Itaituba, Pará, Brasil (Squamata). In Anais do IX Congresso Latino Americano de Herpetologia e V Congresso Brasileiro de Herpetologia. 2011. Curitiba. Curitiba: Sociedade Brasileira de Herpetologia. 
FROTA, JG., SANTOS-JUNIOR AP., CHALKIDIS, HM, and GUEDES, AG., 2005. As serpentes do baixo Rio Amazonas, Oeste do estado do Pará, Brasil (Squamata). Biociencias, vol. 13, p. 211-220.

FUNK, WC., CAMINER, M. and RON, SR., 2011. High level of cryptic species diversity uncovered in Amazonian frogs. Proceedings of the Royal Society B., p. 1-9.

GASCON, C., LOUGHEED, SC. and BOGART, JP., 1998. Patterns of genetic population differentiation in four species of Amazonian frogs: a test of the riverine barrier hypothesis. Biotropica, vol. 30, no. 1, p. 104-119. http://dx.doi.org/10.1111/j.1744-7429.1998. tb00373.x.

HAMMER, O., HARPER, DAT. and RYAN, PD., 2001. PAST: Paleontological statistics software package for education and data analysis. Palaeontologia Electronica, vol. 4, no. 1, p. 1-9.

HILL, MO., 1973. Diversity and evenness: a unifying notation and its consequences. Ecology, vol. 54, no. 2, p. 427-432. http:// dx.doi.org/10.2307/1934352.

HOWLAND, JM., VITT, L. and LOPEZ, PT., 1990. Life on the edge: the ecology and life history of the tropidurine iguanid lizard Uranoscodon superciliosum. Canadian Journal of Zoology, vol. 68, no. 7, p. 1366-1373. http://dx.doi.org/10.1139/z90-204.

JANSEN, M., BLOCH, R., SCHULZE, A. and PFENNINGER, M., 2011. Integrative inventory of Bolivia's lowland anurans reveals hidden diversity. Zoologica Scripta, vol. 40, no. 6, p. 567-583. http://dx.doi.org/10.1111/j.1463-6409.2011.00498.x.

KNISPEL, SR. and BARROS, FB., 2009. Anfíbios anuros da região urbana de Altamira (Amazônia Oriental), Pará, Brasil. Biotemas, vol. 22, no. 2, p. 191-194.

Leme Engenharia, 2009. Aproveitamento hidrelétrico Belo Monte: estudo de impacto ambiental. [s.1.]: Leme Engenharia.

LIMA, AP., MAGNUSSON, WE., MENIN, M., ERDTMANN, LK., RODRIGUES, DJ., KELLER, C., and HÖDL, W., 2006. Guia de sapos da Reserva Adolpho Ducke - Amazônia Central. Manaus: Editora Attema/ INPA. 168 p.

MACEDO, LC., BERNARDE, PS. and ABE, AS., 2008. Lagartos (Squamata: Lacertilia) em áreas de floresta e de pastagem em Espigão do Oeste, Rondônia, sudoeste da Amazônia, Brasil. Biota Neotropica, vol. 8, no. 1, p. 133-139. http://dx.doi.org/10.1590/ S1676-06032008000100016.

MACIEL, AO. and HOOGMOED, MS., 2011. Taxonomy and distribution of caecilian amphibians (Gymnophiona) of Brazilian Amazonia, with a key to their identification. Zootaxa, vol. 2984, p. 1-53.

MACIEL, AO., COSTA, HC., DRUMMOND, LO., GOMES, JO. and D'ANGIOLELLA, A., 2013. Rediscovery of Siphonops annullatus (Mikan, 1820) (Amphibia: Gymnophiona: Siphonopidae) in the State of Pará, Brazil, with an updated geographic distribution map, and notes on size and variation. Check List, vol. 9, no. 1, p. 106-110. http://dx.doi.org/10.15560/9.1.106.

MAGNUSSON, W., BRAGA-NETO, R., PEZZINI, F., BACCARO, F., BERGALLO, H., PENHA, J., RODRIGUES, D.,VERDADE, LM., LIMA, A., ALBERNAZ, AL., HERO, J., LAWSON, B., CASTILHO, C., DRUCKER, D., FRANKLIN, E., MENDONÇA, F., COSTA, F., GALDINO, G., CASTLEY, G., ZUANON, J. VALE, J. SANTOS, JLC., LUIZÃO, R. CINTRA, R., BARBOSA, RI., LISBOA, A., KOBLITZ, RV., CUNHA, CN. and PONTES, ARM., 2013. Biodiversidade e monitoramento ambiental integrado [livro eletrônico] $=$ Biodiversity and integrated environmental monitoring. Santo André: Áttema Editorial. 356 p.

MAGNUSSON, WE., LIMA, AP., LUIZAO, R., LUIZAO, F., COSTA, FRC., CASTILHO, VC. and KINUPP, VF., 2005. RAPELD: A modification of the gentry method for biodiversity surveys in long-term ecological research sites. Biota Neotropica, vol. 5, no. 2, p. 19-24.

MASCHIO, GF., SANTOS-COSTA, MC. and PRUDENTE, AL., 2009. Comunidades de Serpentes da região de Caxiuanã com avaliação da eficiência dos métodos de captura. In LISBOA, PLB. (Org.). Caxiuanã: desafios para a conservação de uma floresta nacional na Amazônia. Belém: Museu Paraense Emílio Goeldi. p. 589-603.

MELO, AS., 2008. O que ganhamos 'confundindo' riqueza de espécies e equabilidade em um índice de diversidade? Biota Neotropica, vol. 8, no. 3, p. 21-27. http://dx.doi.org/10.1590/ S1676-06032008000300001.

MENDES-PINTO, TJ. and SOUZA, SM., 2011. Preliminary assessment of amphibians and reptiles from Floresta Nacional do Trairão, with a new snake record for the Pará state, Brazilian Amazon. Salamandra, vol. 47, no. 4, p. 199-206.

MENDES-PINTO, TJ. and TELLO, JCR., 2010. Répteis Squamata de uma área de transição Floresta-Savana na região Oeste do Estado do Pará, Brasil. Revista de Ciências Ambientais, vol. 4, p. 19-35.

MENIN, M., LIMA, AP., MAGNUSSON, WE. and WALDEZ, F., 2007. Topographic and edaphic effects on the distribution of terrestrially reproducing anurans in Central Amazonia: mesoscale spatial patterns. Journal of Tropical Ecology, vol. 23, no. 5, p. 539-547. http://dx.doi.org/10.1017/S0266467407004269.

NASCIMENTO, HEM. and LAURANCE, WF., 2006. Efeitos de área e de borda sobre a estrutura florestal em fragmentos de floresta de terra-firme após 13-17 anos de isolamento. Acta Amazonica, vol. 36, no. 2, p. 183-192. http://dx.doi.org/10.1590/ S0044-59672006000200008.

OKSANEN, J., BLANCHET, FG., KINDT, R., LEGENDRE, P., MINCHIN, PR., O'HARA, RB., SIMPSON, GL., SOLYMOS, P., STEVENS, MHH. and WAGNER, H., 2013. vegan: Community Ecology Package. The R Project for Statistical Computing. Available from: $<$ http://CRAN.R-project.org/package=vegan $>$. Access in: 21 May 14.

OLIVEIRA, EA., RUZ, EJ. and BARROS, FB., 2013. Herpetofauna de las proximidades de la Caverna Planaltina, Brasil Novo, Pará (Amazonia Brasileña). Herpetotropicos, vol. 9, no. 1-2, p. 55-68.

OLIVEIRA, USC., MENEGHELLI, D. and MESSIAS, MR., 2012. Amphibia, Anura, Centrolenidae, Cochranella adenocheira Harvey and Noonan, 2005: distribution and geographic extension in the state of Rondônia, Brazil. Check List, vol. 8, no. 1, p. 145-146.

PADIAL, JM. and DE LA RIVA, I., 2009. Integrative taxonomy reveals cryptic Amazonian species of Pristimantis (Anura: Strabomantidae). Zoological Journal of the Linnean Society, vol. 155 , no. 1 , p. 97-122. http://dx.doi.org/10.1111/j.10963642.2008.00424.x.

PANTOJA, DL. and FRAGA, R., 2012. Herpetofauna of the Reserva Extrativista do rio Gregório, Amazonas, southwest Brazil. Check List, vol. 8, no. 3, p. 360-374.

PEARSE, DE., ARNDT, AD., VALENZUELA, N., MILLER, BA., CANTARELLI, V. and SITES JUNIOR, JW., 2006. Estimating population structure under nonequilibrium conditions in a conservation context: continent-wide population genetics of the giant Amazon 
river turtle, Podocnemis expansa (Chelonia; Podocnemididae). Molecular Ecology, vol. 15, no. 4, p. 985-1006. http://dx.doi. org/10.1111/j.1365-294X.2006.02869.x. PMid:16599962.

PINHEIRO, LC., BITAR, YOC., GALATTI, U., NECKELOLIVEIRA, S. and SANTOS-COSTA, MC., 2012. Amphibians from southeastern state of Pará: Carajás region, northern Brazil. Check List, vol. 8, no. 4, p. 693-702.

PRUDENTE, ALC. and SANTOS-COSTA, MC., 2005. Checklist of snakes in the Eastern Amazon, Pará State, Brazil: Floresta Nacional de Caxiuanã. Boletim do Museu Paraense Emílio Goeldi, vol. 3 , no. 1, p. 243-251.

PYRON, RA., BURBRINK, FT. and WIENS, JJ., 2013. A phylogeny and revised classification of Squamata, including 4161 species of lizards and snakes. BMC Evolutionary Biology, vol. 13, no. 1, p. 93. http://dx.doi.org/10.1186/1471-2148-13-93. PMid:23627680.

R Development Core Team, 2013. R: A language and environment for statistical computing. Vienna: R Foundation for Statistical Computing. Version 2.13.

ROJAS-MORALES, JA. and ESCOBAR-LASSO, S., 2013. Notes on the natural history of three glass frogs species (Anura: Centrolenidae) from the Andean Central Cordillera of Colombia. Boletín Cientifico Museo de Historia Natural, vol. 17, no. 2, p. $127-140$.

SALOMÃO, RP., VIEIRA, ICG., SUEMITSU, C., ROSA, NA., ALMEIDA, SS., AMARAL, DD. and MENEZES, MPM., 2007. As florestas de Belo Monte na grande curva do rio Xingu, Amazônia Oriental. Boletim do Museu Paraense Emílio Goeldi, vol. 2 , no. 3, p. 57-153.

SILVA, FM., MENKS, AC., PRUDENTE, ALC., COSTA, JCL., TRAVASSOS, AEM. and GALATTI, U., 2011. Squamata reptilies from municipality of Barcarena and surroundings, state of Pará, north of Brazil. Check List, vol. 7, no. 3, p. 220-226.

SIMÕES, PI., KAEFER, IL., GOMES, FBR. and LIMA, AP., 2012. Distribution extension of Hyalinobatrachium cappellei (van Lidth de Jeude, 1904) (Anura: Centrolenidae) across Central Amazonia. Check List, vol. 8, no. 4, p. 636-637.

SOUZA JUNIOR, WC., REID, J. and LEITÃO, NCS., 2006. Custos e beneficios do complexo hidrelétrico Belo Monte: uma abordagem economico-ambiental. 4th ed. Lagoa Santa: CSF. 90 p. Série Técnica.

SOUZA, MB., SILVEIRA, M., LOPES, MRM., VIEIRA, LJS., GUILHERME, E., CALOURO, AM. and MORATO, EF., 2003. Biodiversidade no Estado do Acre: conhecimento atual, conservação e perspectiva. Revista T\&C Amazônia, vol. 1, p. 45-56.
SOUZA, VM., SOUZA, MB. and MORATO, EF., 2008. Efeitos da sucessão florestal sobre a anurofauna (Amphibia: Anura) da Reserva Catuaba e seu entorno, Acre, Amazônia sul-ocidental. Revista Brasileira de Zoologia, vol. 25, no. 1, p. 49-57. http:// dx.doi.org/10.1590/S0101-81752008000100008.

STURARO, MJ. and PELOSO, PLV., 2014. A new species of Scinax Wagner, 1830 (Anura: Hylidae) from the middle Amazon River basin, Brazil. Papéis Avulsos de Zoologia, vol. 54, no. 2, p. 9-23. http://dx.doi.org/10.1590/0031-1049.2014.54.02.

TOLEDO, LF., ARAUJO, OGS., AVILA, RW., KAWASHITARIBEIRO, RA., MORAIS, DH. and CISNEROS-HEREDIA, DF., 2009. Amphibia, Anura, Centrolenidae, Cochranella adenocheira: distribution and range extension, Brazil. Check List, vol. 5, no. 3 , p. $380-382$.

TÓTHMÉRÉSZ, B., 1995. Comparison of different methods for diversity ordering. Journal of Vegetable Science, vol. 6, p. 283-290.

TURCI, LCB. and BERNARDE, PS., 2008. Levantamento herpetofaunístico em uma localidade no município de Cacoal, Rondônia, Brasil. Bioikos, vol. 22, no. 2, p. 101-108.

VAZ-SILVA, W., 2009. Herpetofauna im Zentralbrasilianischen Cerrado. Terraria, vol. 17, p. 75-78.

VITT, LJ and BREITENBACH, GL., 1993. Life histories and reproduction tactics among lizards in the genus Cnemidophorus (Sauria: Teiidae). In WRITHT, JW. and VITT, LJ. (Eds.). Biology of whiptail lizards (genus Cnemidophorus). Norman: Museum of Natural History. p. 211-243.

VITT, LJ. and BLACKBURN, DG., 1991. Ecology and life history of the viviparous lizard Mabuya bistriata (Scincidae) in the Brazilian Amazon. Copeia, vol. 1991, no. 4, p. 916-927. http://dx.doi.org/10.2307/1446087.

VITT, LJ. and ZANI, PA., 1998. Ecological retalionships among sympatric lizards in a transitional forest in the northern Amazon of Brazil. Journal of Tropical Ecology, vol. 14, no. 1, p. 63-70. http://dx.doi.org/10.1017/S0266467498000066.

VITT, LJ., MAGNUSSON, WE., AVILA-PIRES, TC. and LIMA, AP., 2008. Guia de lagartos da Reserva Adolpho Ducke: Amazônia Central. Manaus: Editora Attema/ INPA. 176 p.

VITT, LJ., ZANI, PA. and ÁVILA-PIRES, TC., 1997. Ecology of the arboreal tropidurid lizard Tropidurus (= Plica) umbra in the Amazon region. Canadian Journal of Zoology, vol. 75, no. 11, p. 1876-1882. http://dx.doi.org/10.1139/z97-817.

YANEZ-MUNÕZ, M., PEREZ-PEÑA, P. and CISNEROSHEREDIA, DF., 2009. New country records of Hyalinobatrachium iaspidiense (Amphibia, Anura, Centrolenidae) from the Amazonian lowlands of Ecuador and Peru. Herpetology Notes, vol. 2, p. 49-52. 